\title{
Serum osteopontin and vitronectin levels in systemic sclerosis
}

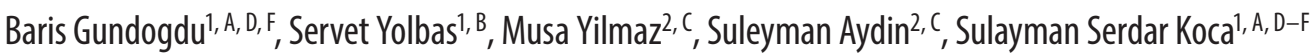 \\ ${ }^{1}$ Department of Rheumatology, Faculty of Medicine, Firat University, Elazığ, Turkey \\ ${ }^{2}$ Department of Biochemistry, Faculty of Medicine, Firat University, Elazığ, Turkey \\ A - research concept and design; $\mathrm{B}$ - collection and/or assembly of data; C - data analysis and interpretation; \\ $D$ - writing the article; $E$ - critical revision of the article; $F$ - final approval of the article
}

Address for correspondence

Sulayman Serdar Koca

E-mail:kocassk@yahoo.com

Funding sources

None declared

\section{Conflict of interest}

None declared

Received on 0ctober 28, 2015

Reviewed on January 9, 2016

Accepted on January 24, 2017
DOI

10.17219/acem/68627

\section{Copyright}

Copyright by Author(s)

This is an article distributed under the terms of the

Creative Commons Attribution Non-Commercial License

(http://creativecommons.org/licenses/by-nc-nd/4.0/)

\section{Abstract}

Background. Osteopontin a matricellular protein has pro-fibrotic effects and binds integrin such as avß1 and av $\beta 3$. Vitronectin is one of the integrin av $\beta 3$ ligands and is a multifunctional glycoprotein.

Objectives. The aim of the present study was to evaluate serum osteopontin and vitronectin levels in a cohort of patients with systemic sclerosis (SSC).

Material and methods. Eighty-six patients with SSc, 46 patients with systemic lupus erythematosus (SLE), and 38 healthy controls (HC) were enrolled in the study. Serum osteopontin, vitronectin, IL-6, and TGF- $\beta$ levels were analyzed.

Results. Serum osteopontin levels were higher in the SSC and SLE groups compared to the HC group $(p<0.01$ and $p<0.001$, respectively). However, it was not correlated with disease activity and severity scores in the SSc group. On the other hand, serum vitronectin levels were lower in the SSc group than in the SLE and HC groups ( $p<0.001$ for both).

Conclusions. These results may suggest that osteopontin levels may be increased due to the inflammatory process and osteopontin has not a specific role on fibrosis in SSc. On the other hand, serum vitronectin levels decrease in SSc in contrast to SLE. It may be concluded that the one cause of decreased serum vitronectin levels in SSc may be its accumulation in fibrotic area.

Key words: systemic sclerosis, osteopontin, vitronectin 


\section{Introduction}

Systemic sclerosis (SSc) is a chronic inflammatory disease, which is characterized by fibrosis in the skin and internal organs. ${ }^{1}$ Its etiopathogenesis is uncertain. However, immune activation and vasculopathy are the main pathogenic issues. The infiltrations of inflammatory cells like $\mathrm{T}$ lymphocytes, mast cells and macrophages are shown in skin biopsies of SSc patients. ${ }^{2}$ Fibrosis occurs with the excessive synthesis and deposition of collagen and other extracellular matrix (ECM) molecules. ${ }^{3}$ Active fibroblasts (myofibroblasts) are responsible for the production of ECM. Although the active mesenchymal cells produce ECM molecules, the source of these cells is controversial. However, the transformation of bone marrow-derived local mesenchymal precursor cells into fibroblastic cells is responsible from the continuity of fibroblastic activity, in SSc. ${ }^{4,5}$

Osteopontin (OPN), which is produced from osteoclasts, osteoblasts, T cells, macrophages, dendritic cells, and fibroblast cells, is a matricelullar glycoprotein, and it has proinflammatory and pro-fibrotic properties. ${ }^{6}$ This molecule modulates signaling between various cells by binding to cell surface integrins $(\alpha v \beta 1, \alpha v \beta 3, \alpha v \beta 5, \alpha v \beta 6, \alpha 5 \beta 1, \alpha 8 \beta 1)$ and to $\mathrm{CD} 44 .^{7,8} \mathrm{OPN}$ stimulates inflammation via the participation of macrophages, dendritic and T-cells into systemic circulation. It contributes to the development of Th1-induced cytokine responses (tumor necrosis factor [TNF]- $\alpha$, interferon- $\gamma$, interleukine [IL]-2), and regulates the behavior of fibroblasts and myofibroblasts differentiation. ${ }^{9-11}$ Furthermore, OPN gene expression has been reported to be increased in SSc. ${ }^{12,13}$ In addition, serum OPN levels are demonstrated to be higher in SSc. ${ }^{14}$ Dermal fibrosis induced by bleomycin has been reported to be less common in mice with OPN gene deficits compared to wild-type mice. ${ }^{15}$

Integrins, a large group of trans-membrane proteins, consist of $\alpha$ and $\beta$ subunits. They mediate cell-cell and cellmatrix interactions. Integrin $\alpha v \beta 3$, the best-known vitronectin (VTN) receptor, consists of $\alpha \mathrm{v}$ (CD51) and $\beta 3$ (CD61) subunits. Although integrin $\alpha v \beta 3$ is expressed at low levels in almost all normal tissues, its expression is high in osteoclasts, neutrophils, macrophages, and endothelial cells. ${ }^{16}$ These cells play an important role in inflammation and angiogenesis, which are pathogenic features of SSc. Moreover, previous studies have demonstrated the contribution of VTN on hepatic, pulmonary and renal fibrosis. ${ }^{16-19}$

The present study aimed to determine the serum levels of OPN and VTN, and also to investigate their probable effects on disease activity and severity indices in SSc.

\section{Material and methods}

\section{Patients and study design}

Eighty-six patients with SSc fulfilling the classification criteria as stipulated by the American College of Rheuma- tology (ACR), 46 patients with systemic lupus erythematosus (SLE) fulfilling classification criteria as stipulated by the Systemic Lupus International collaborating Clinic (SLICC)/ACR, and 38 healthy controls (HC) were enrolled in this study. ${ }^{20-22}$ Local Ethics Committee approved the study, and informed that participant consents were obtained. The rheumatological examination of all participants was also performed. In addition, patients were questioned about clinical manifestations of the disease.

\section{Disease activity and severity scores}

In the SSc group, the extent of skin involvement, degree and severity of internal organ involvement were determined by respectively modified Rodnan skin score (mRSS), Medsger disease severity scale and Valentini disease activity index. ${ }^{23-25}$ Apart from this, the United Kingdom functional score (UKFS) was performed, and fingerpalmar flexion distances were measured. ${ }^{26}$

\section{Laboratory analysis}

Blood samples were taken at 8 am after $8-12$ h of fasting. Routine laboratory, complement (C3-C4) and autoantibody panel tests (ANA-IFA, anti-centromere, anti-Scl-70) were studied on the same day. Erythrocyte sedimentation rate (ESR), C-reactive protein (CRP) levels were assessed by the classic Westergren and immunoturbidimetric methods, respectively. In addition, $5 \mathrm{~mL}$ blood samples were collected for analysis of OPN, IL-6, VTN, TGF- $\beta$, and centrifuged at $5000 \mathrm{rpm}$ for $3 \mathrm{~min}$ to obtain sera. The separated sera were stored at $-20^{\circ} \mathrm{C}$ until use.

OPN (Boster Biological Technology Co., Ltd., Pleasanton, USA), TGF- $\beta$ (Boster Biological Technology Co., Ltd., Pleasanton, USA), IL-6 (Boster Biological Technology Co., Ltd., Pleasanton, USA) and VTN (Eastbiopharm Co., Ltd., Hangzhou, China) levels were measured by the method of enzyme-linked immunosorbent assay (ELISA) using suitable commercial kits.

In the SSc group, pulmonary function test and carbon monoxide diffusing capacity were measured. High-resolution chest computed tomography was performed in patients with abnormal findings on posteroanterior chest $\mathrm{X}$-ray. Pulmonary arterial pressure (PAP) was measured with transthoracic echocardiography and systolic PAP above $40 \mathrm{~mm} \mathrm{Hg}$ was considered as pulmonary arterial hypertension (PAH).

\section{Statistical analysis}

IBM-SPSS 21 software was used in statistical analysis. The data was expressed as mean \pm standard deviation. One-way analysis of variance (ANOVA) and Tukey post-hoc tests were applied for parametric data. Nonparametric data was analyzed using Kruskal-Wallis variance analysis and the Mann-Whitney $U$ test. 
In the parametric data, distribution normality was evaluated with the Kolmogorov-Smirnov test. Logarithmic transformations were applied to the skewed data (ESR, CRP, IL-6, TGF- $\beta$, OPN and VTN) to normalize them before they were entered into the analysis. On the other hand, analysis of covariance (ANCOVA) was also used to adjust variables for age and sex, since mean age and gender distribution were significantly different among the groups. Categorical data was analyzed with the $x^{2}$ test. Pearson correlation analysis was used to assess the relationship between data. P-values $<0.05$ were considered significant.

\section{Results}

Demographics and laboratory data of the study groups are shown in the Table 1 . The mean ages of patients with SSc group were higher compared with SLE and the HC groups ( $\mathrm{p}<0.001$ and $\mathrm{p}<0.05$, respectively). On the other hand, the mean age of patients with SLE group was significantly lower than in the HC group ( $<<0.001)$. Similarly, there were the statistically significant gender differences among the 3 groups ( $\mathrm{p}<0.001$ ). Although female ratios were similar in the SSc and SLE groups ( $p>0.05)$, the male ratios were higher in the $\mathrm{HC}$ group than in SSc and SLE groups ( $\mathrm{p}<0.001$ for both).

In SSc and SLE groups, compared with HC group, ESR levels were higher $(\mathrm{p}<0.001)$. On the other hand, CRP levels in SSc group were significantly higher than the $\mathrm{HC}$ and SLE groups ( $\mathrm{p}<0.05$ and $\mathrm{p}<0.001$, respectively).

Compared with the HC group, serum OPN levels in SSc and SLE groups were statistically significantly higher $(\mathrm{p}<0.01$ and $\mathrm{p}<0.001$, respectively). The increase

Table 1. Demographic and laboratory characteristics of the study groups

\begin{tabular}{|c|c|c|c|c|}
\hline Parameters & $\operatorname{SSC}(n=86)$ & SLE $(n=46)$ & $H C(n=38)$ & $p$-value \\
\hline Age (years) & $51.2 \pm 12.7^{x \times x, t}$ & $34.3 \pm 9.9^{x}$ & $44.2 \pm 14.2$ & $<0.001^{\#}$ \\
\hline Gender (f/m) & $80 / 6^{x}$ & $42 / 4^{x}$ & $20 / 18$ & $<0.001^{\&}$ \\
\hline BMI $\left(\mathrm{kg} / \mathrm{m}^{2}\right)$ & $26.1 \pm 4.7$ & $24.2 \pm 5.1^{x}$ & $27.9 \pm 4.7$ & $0.002^{\#}$ \\
\hline Disease duration (years) & $6.2 \pm 5.1$ & $5.5 \pm 5.3$ & - & 0.453 \\
\hline Leukocyte $\left(10^{3} / \mathrm{mm}^{3}\right)$ & $7.6 \pm 2.6^{\dagger}$ & $5.6 \pm 2.3$ & $6.7 \pm 1.8$ & $<0.001^{\#}$ \\
\hline Thrombocyte $\left(10^{3} / \mathrm{mm}^{3}\right)$ & $312.8 \pm 90.1^{1+t}$ & $260.4 \pm 113.5^{x \times x}$ & $312.8 \pm 92.5$ & $0.009^{\#}$ \\
\hline Hemoglobin (g/dL) & $12.6 \pm 1.6^{x \times, t \dagger}$ & $11.6 \pm 1.9^{x}$ & $13.8 \pm 1.7$ & $<0.001^{\#}$ \\
\hline $\mathrm{ESR}(\mathrm{mm} / \mathrm{h})$ & $27.8 \pm 16.8^{x}$ & $39.1 \pm 28.9^{x}$ & $18.1 \pm 15.8$ & $<0.001^{*}$ \\
\hline CRP (mg/dL) & $1.73 \pm 3.84^{\times x \times, t}$ & $0.74 \pm 1.32$ & $0.93 \pm 2.72$ & $0.002^{*}$ \\
\hline IL-6 (pg/mL) & $22.9 \pm 59.4$ & $16.8 \pm 54.1^{x \times x}$ & $5.3 \pm 3.3$ & $0.234^{*}$ \\
\hline TGF- $\beta$ (pg/mL) & $41.1 \pm 109.7^{x \times,+\dagger}$ & $15.3 \pm 20.6$ & $23.1 \pm 52.3$ & $0.002^{*}$ \\
\hline OPN (ng/mL) & $26.67 \pm 15.33^{x x, t+}$ & $36.86 \pm 18.62^{x}$ & $19.56 \pm 6.41$ & $<0.001^{*}$ \\
\hline VTN (ng/L) & $252.6 \pm 169.1^{x, \dagger}$ & $501.4 \pm 487.7$ & $526.1 \pm 357.2$ & $<0.001^{*}$ \\
\hline
\end{tabular}

SSC - systemic sclerosis; SLE - systemic lupus erythematosus; HC - healthy control; M - male; F - female; $\mathrm{BMI}$ - body mass index; ESR - erythrocyte sedimentation rate; CRP - C-reactive protein; IL - interleukin; TGF- $\beta$ - transforming growth factor-beta; OPN - osteopontin; VTN - vitronectin. Compared with the HC group; ${ }^{x} p<0.001,{ }^{x \times} p<0.01,{ }^{x \times x} p<0.05$. Compared with the SLE group; ${ }^{\dagger} p<0.001,{ }^{+\dagger} p<0.01,{ }^{+\dagger+} p<0.05$. *Kruskal Wallis variance analysis ${ }^{*}$ One-way ANOVA and ${ }^{\&} x^{2}$ test $p$-values. of OPN level remained significant even after adjustments for age and gender (ANCOVA $\mathrm{p}<0.01$ and $\mathrm{p}<0.001$, respectively). However, serum OPN levels in the SSc group were significantly lower than in the SLE group ( $\mathrm{p}<0.05$ and ANCOVA p < 0.05) (Table 1).

Serum VTN levels were significantly lower in the SSc group compared with the SLE and HC groups ( $\mathrm{p}<0.001$ for both). The decrease of VTN level remained significant even after adjustments for age and gender (ANCOVA $\mathrm{p}<0.001$ for both). Serum VTN levels in the SLE and $\mathrm{HC}$ groups were similar ( $\mathrm{p}>0.05$ and ANCOVA $\mathrm{p}>0.05$ ) (Table 1).

In the SSc group, there was no statistically significant difference between females and males in terms of OPN, VTN, IL-6 and TGF- $\beta$ levels ( $p>0.05$ ). Serum TGF- $\beta$ levels were lower in patients receiving glucocorticoids compared to nonusers in the SSc group (33.01 \pm 32.79 vs $44.69 \pm 129.90 \mathrm{pg} / \mathrm{mL}, \mathrm{p}=0.047)$. Similarly, TGF- $\beta$ levels were determined to be lower in patients who receive methotrexate compared to nonusers in the SSc group $(4.74 \pm 8.40$ vs $47.33 \pm 121.13 \mathrm{pg} / \mathrm{mL}, \mathrm{p}=0.046)$. In addition, VTN levels were found to be significantly lower in patients who take methotrexate compared to not taking methotrexate $(189.17 \pm 82.59$ vs $267.13 \pm 180.53 \mathrm{ng} / \mathrm{L}$, $\mathrm{p}=0.034)$.

Clinical characteristics and laboratory data of diffuse cutaneous SSc (dcSSc) and limited cutaneous SSc (lcSSc) subgroups were shown in the Table 2. There was no relationship between the levels of OPN, VTN, IL- 6 and TGF- $\beta$, and the positive ANA, anti-centromere and antiScl-70 in the SSc group ( $\mathrm{p}>0.05$ for all). In the patients with $\mathrm{PAH}$, digital ulcer, internal organ-system involvement such as lungs, cardiovascular system, kidneys and gastrointestinal tract compared with those who do not have these kind of complications, there was no significant difference in terms of OPN, VTN, IL- 6 and TGF- $\beta$ levels ( $p>0.05$ for all). Furthermore, SSc subtypes, the degree of nailfold capillaroscopy findings and Medsger (skin-lung-heart) disease severity index were not related with the levels of OPN, VTN, IL- 6 and TGF- $\beta$ ( $p>0.05$ for all). However, in the SSc group, there was a significant correlation between OPN levels and patients' age $(\mathrm{r}=0.224, \mathrm{p}<0.05)$.

Serum IL-6 level was also correlated with visual analog scale (VAS) score $(\mathrm{r}=0.292, \mathrm{p}<0.01)$. On the other hand, there was negative correlation between OPN and VTN levels in the SSc group $(\mathrm{r}=-0.269, \mathrm{p}<0.05)$. 


\section{Discussion}

In this study, serum OPN and VTN levels were studied in patients with SSc and SLE. Serum OPN levels were higher in SLE as well as SSc in the present study. On the other hand the serum VTN level was decreased in SSc in contrast to SLE.

Systemic sclerosis is a chronic systemic disease characterized by the fibrosis of the skin and internal organs. Although the etiopathogenetic mechanism of SSc is not fully understood, the pathogenesis of SSc consists of a triad including fibrosis, vasculopathy and inflammation. ${ }^{2}$ Fibrosis occurs as a result of excessive ECM production or insufficient ECM degradation. ${ }^{3}$ Fibroblasts, which are responsible in the production of ECM, are the main actors in the pathogenesis of SSc. In this process, the pro-fibrotic cytokines and chemokines are responsible in the transformation and proliferation of myofibroblasts. ${ }^{4,5}$ Also, these mediators are released from inflammatory cells and activated and/or damaged endothelial cells. It is known that the inflammation and vasculopathy as pathogenetic stages of SSc begin the adventure before fibrosis. ${ }^{1}$ The infiltration of inflammatory cells such as $\mathrm{T}$ lymphocytes,

Table 2. Disease activity and severity scores, organ system involvements and laboratory features in the subtypes of patients with SSC

\begin{tabular}{|l|c|c|c|}
\multicolumn{1}{|c|}{ Parameters } & $\begin{array}{c}\text { dcSSc } \\
(n=12)\end{array}$ & IcSSc $(\mathrm{n}=67)$ & p-value \\
\hline ANA positivity, $n$ (\%) & $12(100)$ & $59(88.1)$ & $0.207^{\times}$ \\
\hline $\begin{array}{l}\text { Anti-centromere positivity, } \\
\mathrm{n}(\%)\end{array}$ & $1(8.3)$ & $11(16.4)$ & $0.418^{\times}$ \\
\hline Anti-Scl 70 positivity, n (\%) & $12(100)$ & $29(43.3)$ & $<0.001^{\times}$ \\
\hline mRSS & $18.7 \pm 7.1$ & $9.9 \pm 5.1$ & $<0.001^{*}$ \\
\hline Valentine disease activity & $1.3 \pm 0.5$ & $1.6 \pm 0.5$ & $0.059^{*}$ \\
\hline index & $1.7 \pm 0.8 /$ & $1.2 \pm 0.4 /$ & $0.001 /$ \\
\hline Medsger disease severity & $1.3 \pm 0.5$ & $0.8 \pm 0.9$ & $0.023^{*}$ \\
\hline SCale (skin/pulmonary) & $5.4 \pm 1.7$ & $5.1 \pm 1.6$ & $0.525^{*}$ \\
\hline VAS for patients & $6.3 \pm 0.6$ & $4.7 \pm 1.6$ & $0.001^{*}$ \\
\hline VAS for physicians & $4.2 \pm 4.3$ & $6.1 \pm 8.6$ & $0.641^{*}$ \\
\hline UKFS & $12(100)$ & $31(46.3)$ & $<0.001^{\times}$ \\
\hline ILD, n (\%) & $5(41.7)$ & $14(20.9)$ & $0.893^{\times}$ \\
\hline PAH, n (\%) & $29.3 \pm 14.4$ & $27.2 \pm 17.1$ & $0.512^{*}$ \\
\hline ESR (mm/h) & $1.2 \pm 1.7$ & $1.8 \pm 4.3$ & $0.552^{*}$ \\
\hline CRP (mg/dL) & $32.5 \pm 68.7$ & $20.7 \pm 60.1$ & $0.299^{*}$ \\
\hline IL-6 (pg/mL) & $105.1 \pm 260.8$ & $31.1 \pm 46.3$ & $0.291^{*}$ \\
\hline TGF- $\beta$ (pg/mL) & $30.5 \pm 17.1$ & $26.6 \pm 15.4$ & $0.325^{*}$ \\
\hline OPN (ng/mL) & $210.2 \pm 92.5$ & $261.9 \pm 183.1$ & $0.390^{*}$ \\
\hline VTN (ng/L) & & & \\
\hline
\end{tabular}

dcSSC - diffuse cutaneous systemic sclerosis; ICSSC - limited cutaneous systemic sclerosis; ANA - anti-nuclear antibody; VAS - visual analog scale; UKFS - United Kingdom functional score; ILD - interstitial lung disease; $\mathrm{PAH}$ - pulmonary arterial hypertension; ESR - erythrocyte sedimentation rate; CRP - C-Reaktif protein; IL - interleukin; TGF- $\beta$ - transforming growth factor-beta; OPN - osteopontin; VTN - vitronectin. *Mann-Whitney U test, and $\times{ }^{2}$ test $p$-values mast cells and macrophages has been shown in the skin biopsies of patients with SSc. ${ }^{1-4}$

Osteopontin, a pleiotropic cytokine, participates in the recruitment and retention of inflammatory cells such as T lymphocytes, macrophages to the site of inflammation. ${ }^{8-10}$ In addition to its possible effects on the stage of inflammation in the pathogenesis of SSc, OPN directly regulates the fibroblastic activity ${ }^{11}$ In this regard, the 1.4fold increase compared to patients with idiopathic PAH, and 4-fold increase compared to healthy volunteers in the serum OPN levels of SSc patients were observed in a previous clinical study. ${ }^{14}$ Subsequently, in another study, serum OPN levels have been determined to be higher in patients with SSc compared to the HC group. ${ }^{13}$ Similarly, in our study, serum OPN levels were higher in the SSc group.

Because of its level being higher in SSc and SLE groups in this study, the increase in the level of OPN in SSc was thought to be associated with the inflammatory nature of the disease. It has been shown that the classic mediators of acute inflammation such as TNF- $\alpha$ and IL- $1 \beta$ induce the OPN expression strongly, as well as the fibrogenic cytokines such as TGF- $\beta$ and angiotensin II can also induce OPN upregulation. ${ }^{27}$ OPN expressed by bone and kidney in physiological conditions is known to be expressed through inflammatory cells, especially $\mathrm{T}$ cells, macrophages and dendritic cells in pathological conditions. ${ }^{6}$

In the present study, the fact that there is no correlation between serum OPN levels and the amount of fibrosis or disease severity supports the view that OPN is not directly associated with fibrosis. On the other hand, Lorenzen et al. have reported that the level of OPN is higher in patients with dcSSc than in lcSSc in whom the patients with former subtype have wider fibrosis. ${ }^{14}$ It has also been reported in the same study that there is a correlation between OPN levels and mRSS. Moreover, OPN levels have been showed to be higher in patients with $\mathrm{PAH}$ and interstitial lung disease (ILD) compared to non-PAH and ILD patients. ${ }^{14}$ One reason for not verifying these relations in this study may be the heterogeneous nature of the disease. The inflammatory state can be variable at different stages of the disease and even in different visceral involvements.

It has been demonstrated previously that the levels of OPN are elevated in various inflammatory diseases. ${ }^{28-30}$ In the present study, the relationship between serum OPN levels and SSc subtypes, disease activity, and severity scores were not detected. In addition, there was no correlation between serum OPN levels and laboratory parameters specific to SSc and capillaroscopy findings. According to these findings, it can be concluded that as OPN does not have a directly pathogenic role in SSc, and its levels rise indirectly due to the inflammatory nature of the disease.

Another important ECM protein is VTN as in OPN. VTN, one of the integrin $\alpha v \beta 3$ ligands, is a multifunctional 
glycoprotein that is present in blood and in the ECM. ${ }^{31}$ This protein, which is an adhesive glycoprotein in structure, is initially defined as serum spreading factor in blood. VTN plays an important role in fibrinolysis, the immune defense, and hemostasis by providing cell adhesion and migration through interaction with collagen. ${ }^{32-34}$ In the present study, serum VTN levels were detected lower in SSc group. The one reason of the decreased VTN levels may be that it functions as a negative acute phase reactant. The decreased levels of VTN have been reported in patients with rheumatoid arthritis and Behçet's disease in another study. ${ }^{35}$ However, in the current study, the undiminished levels of VTN in SLE, which is another inflammatory disease, and the lack of association of serum VTN levels with the levels of cytokines and acute phase reactants in both SSc and SLE may challenge this argument.

In contrast to SLE, VTN levels being reduced in SSc may cause another interpretation that VTN has anti-fibrotic effects, and reduced VTN level augments fibrosis in SSc. While binding to $\alpha v \beta 3$ integrin via Arg-Gly-Asp (RGD) domain, VTN binds to Urokinase-type plasminogen activator receptor (uPAR) by somatomedin B domain. ${ }^{19}$ A more recent study demonstrates that inactivation of UPAR gene induces dermal and pulmonary fibrosis in mice. ${ }^{36}$ Considering the relationship between VTN with UPAR, VTN could be interpreted as anti-fibrotic potential. However, the delayed wound healing in response to tissue damage and decreased angiogenesis has been reported to be associated with the deficiency of VTN in mice. ${ }^{37}$ In the literature, the relatively raised accumulation of VTN in tissues has been reported to be associated with fibrosis of internal organs, such as liver, lungs and kidneys. ${ }^{17-19}$ In subsequent studies, the increased expression of integrin $\alpha v \beta 5$, which serves as a receptor for VTN by SSc dermal fibroblasts, and the role of VTN in stimulating the autocrine TGF- $\beta$ signaling pathway in dermal fibroblasts derived from localized SSc have been demonstrated. ${ }^{38,39}$ According to this data, it may be accepted that VTN does not have anti-fibrotic effects. Then, the most probable explanation of the decreased serum levels of VTN in patients with SSc is the accumulation of VTN in fibrotic skin and subcutaneous tissues. Lopez-Guize et al. have showed the accumulation of VTN in the experimental models of renal fibrosis in fibrotic areas. ${ }^{19}$

There are some limitations of this study. Foremost, the levels of OPN and VTN were only investigated in serum. In addition to serum levels, the assessment of local tissue expression could be more accurate. Moreover, the sample size is relatively small.

As a result, OPN levels are increased in chronic autoimmune diseases such as SLE and SSc. Contrary to common belief, OPN may not be a specific cytokine involved in the stage of fibrogenesis in SSc. This molecule plays a role possibly as part of the acute inflammatory response by regulating the chemotaxis of macrophages and T cells towards the area of inflammation. On the other hand, serum VTN levels decrease in SSc in contrast to SLE and it may be concluded that VTN has anti-fibrotic action or the one cause of decreased serum VTN levels in SSc may be the accumulation of this ECM protein in fibrotic area.

\section{References}

1. Varga J, Abraham D. Systemic sclerosis: A prototypic multisystem fibrotic disorder. J Clin Invest. 2007;117:557-567.

2. Rosenbloom J, Castro SV, Jimenez SA. Narrative review: Fibrotic diseases: Cellular and molecular mechanisms and novel therapies. Ann Intern Med. 2010;152:159-166.

3. Denton CP, Black CM, Abraham DJ. Mechanisms and consequences of fibrosis in systemic sclerosis. Nat Clin Pract Rheumatol. 2006;2:134-144.

4. Krieg T, Abraham D, Lafyatis R. Fibrosis in connective tissue disease: The role of the myofibroblast and fibroblast-epithelial cell interactions. Arthritis Res Ther. 2007;9(Suppl 2).

5. Postlethwaite $A E$, Shigemitsu $H$, Kanangat S. Cellular origins of fibroblasts: Possible implications for organ fibrosis in systemic sclerosis. Curr Opin Rheumatol. 2004;16:733-738.

6. Wang KX, Denhardt DT. Osteopontin: Role in immune regulation and stress responses. Cytokine Growth Factor Rev. 2008;19:333-345.

7. Anborgh PH, Mutrie JC, Tuck AB, Chambers AF. Pre- and post-translational regulation of osteopontin in cancer. J Cell Commun Signal. 2011;5:111-122.

8. Rittling SR. Osteopontin in macrophage function. Exp Rev Mol Med. 2011;13:e15.

9. Ashkar S, Weber GF, Panoutsakopoulou V, et al. Eta-1 (osteopontin): An early component of type-1 (cell-mediated) immunity. Science. 2000;287:860-864.

10. Zheng W, Li R, Pan $H$, et al. Role of osteopontin in induction of monocyte chemoattractant protein 1 and macrophage inflammatory protein 1beta through the NF kappaB and MAPK pathways in rheumatoid arthritis. Arthritis Rheum. 2009;60:1957-1965.

11. Lenga Y, Koh A, Perera AS, McCulloch CA, Sodek J, Zohar R. Osteopontin expression is required for myofibroblast differentiation. Circ Res. 2008;102:319-327.

12. Gardner H, Shearstone JR, Bandaru R, et al. Gene profiling of scleroderma skin reveals robust signatures of disease that are imperfectly reflected in the transcript profiles of explanted fibroblasts. Arthritis Rheum. 2006;54:1961-1973.

13. Barizzone N, Marchini M, Cappiello F, et al. Association of osteopontin regulatory polymorphisms with systemic sclerosis. Hum Immunol. 2011;72:930-934.

14. Lorenzen JM, Krämer R, Meier M, et al. Osteopontin in the development of systemic sclerosis-relation to disease activity and organ manifestation. Rheumatology (Oxford). 2010;49:1989-1991.

15. Wu M, Schneider DJ, Mayes MD, et al. Osteopontin in systemic sclerosis and its role in dermal fibrosis. J Invest Dermatol. 2012;132: 1605-1614.

16. Horton MA. The alpha v beta 3 integrin "vitronectin receptor". Int J Biochem Cell Biol. 1997;29:721-725.

17. Chen W, Rock JB, Yearsley MM, Ferrell LD, Frankel WL. Different collagen types show distinct rates of increase from early to late stages of hepatitis C-related liver firbosis. Hum Pathol. 2014;45:160-165.

18. Carpagnano GE, Kharitonov SA, Wells AU, Pantelidis P, Du Bois RM, Barnes PJ. Increased vitronectin and endotelin-1 in the breath condensate of patients with fibrosing lung disease. Respiration. 2003;70:154-160.

19. López-Guisa JM, Rassa AC, Cai X, Collins SJ, Eddy AA. Vitronectin accumulates in the interstitium but minimally impacts fibrogenesis in experimental chronic kidney disease. Am J Physiol Renal Physiol. 2011;300:1244-1254.

20. Subcommittee for scleroderma criteria of the American Rheumatism Association Diagnostic and Therapeutic Criteria Committee. Preliminary criteria for the classification of systemic sclerosis (scleroderma). Arthritis Rheum. 1980;23:581-590.

21. van den Hoogen F, Khanna D, Fransen J, et al. 2013 Classification criteria for systemic sclerosis: An American College of Rheumatology/European League Against Rheumatism Collaborative Initiative. Ann Rheum Dis. 2013;72:1747-1755. 
22. Petri M, Orbai AM, Alarcón GS, et al. Derivation and validation of the Systemic Lupus International Collaborating Clinics classification criteria for systemic lupus erythematosus. Arthritis Rheum. 2012;64:2677-2686.

23. Clements $P$, Lachenbruch $P$, Siebold J, et al. Inter and intraobserver variability of total skin thickness score (modified Rodnan TSS) in systemic sclerosis. J Rheumatol. 1995;22:1281-1285.

24. Medsger TA Jr, Silman AJ, Steen VD, et al. A disease severity scale for systemic sclerosis: Development and testing. J Rheumatol. 1999:26:2159-2167.

25. Valentini G, Della Rossa A, Bombardieri S, et al. European multicentre study to define disease activity criteria for systemic sclerosis. II. Identification of disease activity variables and development of preliminary activity indexes. Ann Rheum Dis. 2001;60:592-598.

26. Fries JF, Spitz PW, Young DY. The dimensions of health outcomes: The Health Assesment Questionnaire, disability and pain scales. J Rheumatol. 1982;9:789-793.

27. Mazzali M, Kipari T, Ophascharoensuk V, Wesson JA, Johnson R, Hughes J. Osteopontin - A molecule for all seasons. QJM. 2002;95:3-13.

28. Rullo OJ, Woo JM, Parsa MF, et al. Plasma levels of osteopontin identify patients at risk for organ damage in systemic lupus erythematosus. Arthritis Res Ther. 2013;15(1):R18.

29. Ji HI, Lee SH, Song R, et al. Serum level of osteopontin as an inflammatory marker does not indicate disease activity or responsiveness to therapeutic treatments in patients with rheumatoid arthritis. Clin Rheumatol. 2014;33(3):397-402.

30. Erturkler E, Cicek D, Kaman D, Ozdogan S, Bakar Dertlioglu S. Plasma osteopontin levels in patients with Behcet's disease and psoriasis. Eur J Dermatol. 2011;21:203-208.

31. Schvartz I, Seger D, Shaltiel S. Vitronectin. Int J Biochem Cell Biol. 1999;31:539-544.

32. Hayman EG, Pierschbacher MD, Ohgren Y, Ruoslahti E. Serum spreading factor (vitronectin) is present at the cell surface and in tissues. Proc Natl Acad Sci USA. 1983;80:4003-4007.

33. Mayasundari A, Whittemore NA, Serpersu EH, Peterson CB. The solution structure of the N-terminal domain of human vitronectin: Proximal sites that regulate fibrinolysis and cell migration. J Biol Chem. 2004;279:29359-29366.

34. Preissner KT. The role of vitronectin as multifunctional regulator in the hemostatic and immune systems. Blut. 1989;59:419-431.

35. Koca SS, Kara M, Ozgen M, et al. The rs3768777-G allele of ITGAV gene is associated with rheumatoid arthritis. Rheumatol Int. 2014;34:693-698.

36. Manetti M, Rosa I, Milia AF, et al. Inactivation of urokinase-type plasminogen activator receptor (UPAR) gene induces dermal and pulmonary fibrosis and peripheral microvasculopathy in mice: A new model of experimental scleroderma? Ann Rheum Dis. 2014;73:1700-1709.

37. Jang YC, Tsou R, Gibran NS, Isik FF. Vitronectin deficiency is associated with increased wound fibrinolysis and decreased microvascular angiogenesis in mice. Surgery. 2000;127:696-704.

38. Asano Y, Ihn H, Yamane K, Kubo M, Tamaki K. Increased expression levels of integrin alphavbeta5 on scleroderma fibroblasts. Am J Pathol. 2004;164:1275-1292.

39. Asano $Y$, Ihn $H$, Jinnin $M$, Mimura $Y$, Tamaki $K$. Involvement of alphavbeta5 integrin in the establishment of autocrine TGF- $\beta$ signaling in dermal fibroblasts derived from localized scleroderma. J Invest Dermatol. 2006;126:1761-1769. 